\title{
ORIGINAL ARTICLE \\ Sepsis of the hip due to pressure sore in spinal cord injured patients: advocacy for a one-stage surgical procedure
}

\author{
M Le Fort ${ }^{1}$, J Rome-Saulnier ${ }^{1}$, F Lejeune ${ }^{2}$, F Bellier-Waast ${ }^{2}$, S Touchais $^{3}$, P Kieny ${ }^{1}$, F Duteille ${ }^{2}$ and \\ B Perrouin-Verbe ${ }^{1}$
}

Study design: Retrospective study reporting characteristics and management of septic arthritis of the hip due to pressure sores in spinal cord-injured patients.

Objectives: To describe clinical and biological data of septic arthritis of the hip and its treating management.

Setting: The database of the regional $\mathrm{SCl}$ referral center, Nantes, France.

Methods: We retrospectively collected data from 33 cases of septic arthritis of the hip in the medical files of 26 patients.

Results: We analyzed 33 cases of septic arthritis of the hip treated in one French referent center for spinal cord-injured patients from January 1988 to December 2009. Most patients had a thoracic complete paraplegia and nearly two-third (17 out of 26) had no systematic follow-up. In 25 out of 33 cases, the septic arthritis of the hip was due to a trochanteric pressure sore. The causal pressure sore was most frequently associated with a persistent drainage. The standard radiological examination led to the diagnosis in 30 cases and, in 7 questionable cases, magnetic resonance imaging was more contributory. Surgery always consisted of a wide carcinologicallike excision and of a subtrochanteric proximal femoral resection including both greater and lesser trochanters. A musculocutaneous flap was realized for all cases and the choice of the muscle depended on the localization of the causal pressure sore but also of the remaining choices, as most of the patients had already undergone a prior surgery. An antibiotic treatment was adapted to multiple samples during surgery.

Conclusion: We do advocate for a one-stage procedure including a subtrochanteric proximal femoral resection and a musculocutaneous flap.

Spinal Cord (2015) 53, 226-231; doi:10.1038/sc.2014.170; published online 4 November 2014

\section{INTRODUCTION}

It is estimated that up to $80 \%$ of patients with spinal cord injury (SCI) will experience at least one pressure sore during their lifetime, ${ }^{1}$ leading to a reduction of their social participation and also to astounding medical and economic impacts on health systems. ${ }^{2}$ Septic contamination of the hip from a nearby pressure sore is a distressing complication, whose gold standard treatment has been discussed in the literature, with some variations in management. We report here on the experience of the French SCI referral center of Nantes University Hospital with the aim of describing the clinical and bacteriological profile of septic arthritis in SCI patients and to discuss and then to suggest a convenient management method of this life-threatening complication.

\section{PATIENTS AND METHODS}

This retrospective survey involved 26 patients ( 20 men and 6 women) with a mean age of 39 years $(23-66$; s.d. $=13.5)$ and a post-injury delay of 14.5 years (2.5-39; s.d. = 5.16). The spinal cord lesion was post-traumatic for 21 patients. The other etiologies were multiple sclerosis (MS; 3 cases), ischemic myelitis ( 1 case) and primary syringomyelia ( 1 case). Thirty-three sepses of the hip (SH), always due to pelvic pressure sores, were treated between 1988 and 2009 in a French SCI referral center. All the patients were hospitalized in one specific
SCI unit of the PRM (Physical and Rehabilitation Medicine) department for a few days before surgery to evaluate the intended anesthetic and surgical procedures and to prepare for the operation. All the patients were returned from the plastic surgery department to the specialized PRM unit on the same day as the surgery or, at the latest, the next day. During the postoperative period, the patients lay supine on a low-air-loss bed. We used $3 \mathrm{~kg}$ skeletal bonded traction over 3 weeks. The suction drains were monitored several times a day; the sutures were removed after 21 days. Four weeks of permanent hip extension were managed with pillows and sheets in bed. Then, hip flexion postures were started and increased day by day. A gradual return to the sitting position in the wheelchair began after 6 weeks of confinement.

The medical files were reviewed to record notable clinical and bacteriological characterizations, the type of surgical procedure and the occurrence of secondary complications.

We certify that all applicable institutional and governmental regulations concerning the ethical use of human volunteers' medical files were followed during the course of this research.

\section{RESULTS}

Neurological status

According to the International Standards for Neurological Classification of Spinal Cord Injury, 21 of 26 lesions were classified as American Spinal Injury Association (ASIA) Impairment Scale (AIS) grade A

${ }^{1}$ Neurological Physical Medicine and Rehabilitation Department, University Hospital, Hôpital Saint-Jacques, Nantes Cedex 01, France; ${ }^{2}$ Burnt Persons and Plastic Surgery Department, University Hospital - Hôtel-Dieu, Nantes Cedex 01, France and ${ }^{3}$ Orthopaedic Surgery Department, University Hospital - Hôtel-Dieu, Nantes Cedex 01, France Correspondence: Dr M Le Fort, Neurological Physical Medicine and Rehabilitation Department, University Hospital of Nantes, Hopital Saint-Jacques, 85 rue Saint-Jacques, 44093 Nantes Cedex 01, France.

E-mail: marc.lefort@chu-nantes.fr

Received 6 April 2014; revised 8 August 2014; accepted 27 August 2014; published online 4 November 2014 


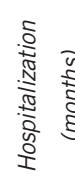

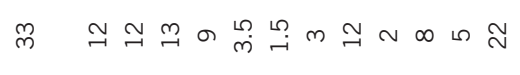

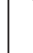

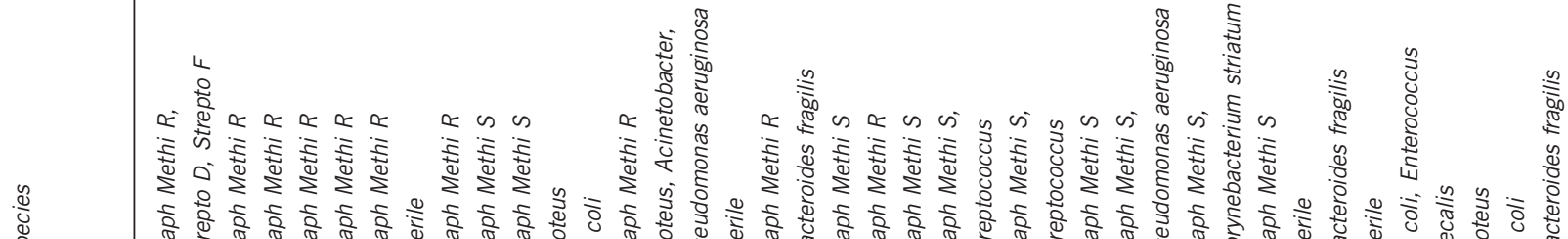
至

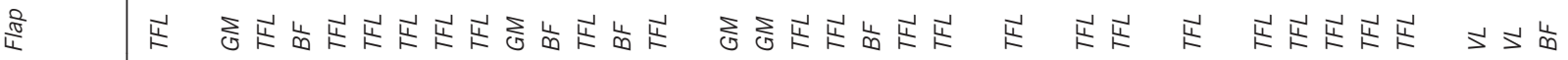

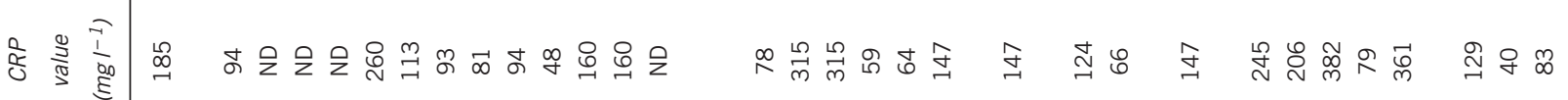

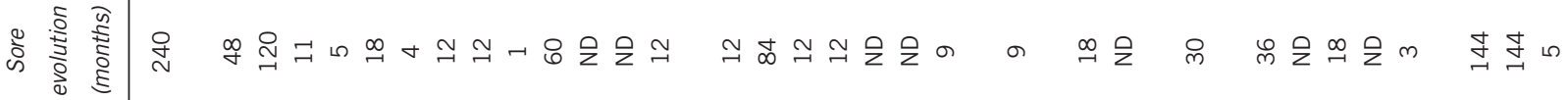

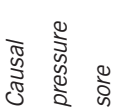
ミートトトトトトトー

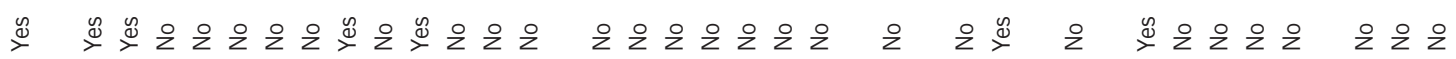

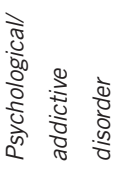

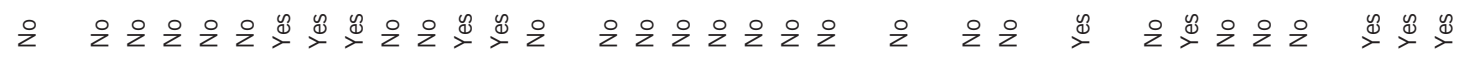

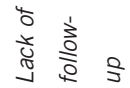
(⿻)

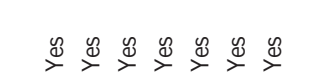
$\stackrel{0}{2} \stackrel{\infty}{>}$

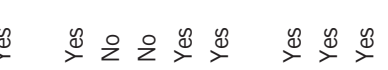

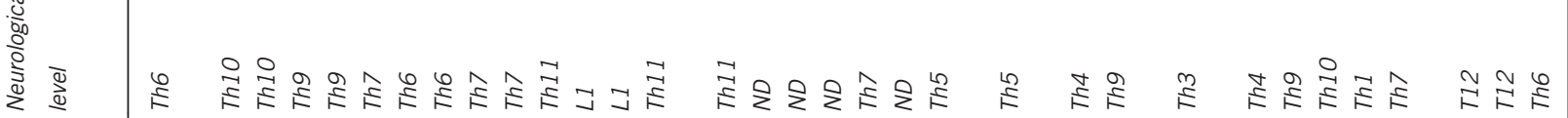
ॐे $\pi$

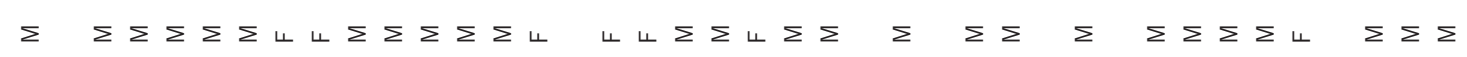


(complete SCI), and 2 were classified as AIS grade B (incomplete sensory) (Table 1). The neurological level was thoracic in 22 patients and was lumbar in 1 patient. These data were not determined for the three patients with MS. The clinical presentation was spastic in 19 patients and flaccid in 7 patients.

\section{Specific risk factors}

No systematic follow-up was noted in 17 of 26 patients. Two other risk factors were psychiatric and/or addictive disorders in eight cases and arterial disease in six

\section{Characteristics of pelvic pressure sores and sepses of the hip} The localization of the pressure sore associated with an $\mathrm{SH}$ was trochanteric in 25 cases and ischial in $8 ; 7$ cases of bilateral SH occurred with any possible combination of pressure sores. Of the 26 cases of SH for which this information was available, the offending pressure sore had been evolving for a mean duration of 46 months $(1-240$; s.d. $=61)$ and had already been treated at least once in 16 cases $(61.5 \%)$. Prior treatment of the SH had also been noted in 10 cases and consisted of insufficient femoral shaft resection, always performed in French non-referral centers. Twenty-two patients presented with multiple sores at the time of the SH.

The diagnosis of SH was clinically evident in four cases of hip exposure and was highly suspected in cases of hip dislocation in five patients with a history of pelvic pressure sores and in five patients with persistent drainage after femoral resection. Otherwise, the most frequent clinical presentation was a stage 4 trochanteric or ischial pressure sore, according to the National Pressure Ulcer Advisory Panel staging, with persistent and heavy drainage (17 of $33 \mathrm{SH}$ ). Two cases of SH were diagnosed only during the surgical procedure, whereas the diagnosis had already been confirmed by radiological and biological data before surgery in all of the other cases. The standard radiological examination likely led to a diagnosis in 30 cases, showing femoral head and neck lysis, erosion of the joint surface and/or a dislocation of the hip (Figure 1). In seven questionable cases, MRI (magnetic resonance imaging) was the most contributory examination that showed edema of the bones with low signal T1-weighted and high signal T2-weighted images (Figure 2), synovitis and overall effusion inside the joint space. The blood tests showed inflammation, based on the C-reactive protein levels, with the highest value being greater than $50 \mathrm{mgl}^{-1}$ in $93 \%$ (26 of 28 known) and greater than $100 \mathrm{mgl}^{-1}$ in $57 \%$ (16 of 28 known) of our cases.

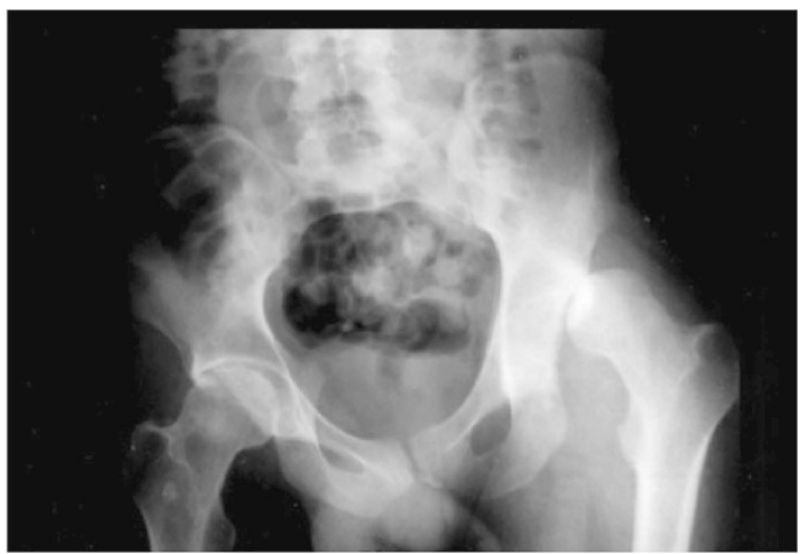

Figure 1 Septic dislocation of the left hip.
Surgical procedures and bacteriological data

Proximal femoral resection. Surgery always consisted of wide oncological-like excision of all necrotic tissues and subtrochanteric proximal femoral resection (Figure 3), including removal of both the greater and lesser trochanters. This procedure was always combined with a capsulectomy and acetabular reaming, and corrected prior insufficient resection in 10 cases.

Tissue coverage. Closure was realized by a musculocutaneous flap for the 33 cases of SH. The entire surgical procedure, proximal femoral resection and flap, was always performed in one stage. Multiple drains with large diameters allowed for closed suction drainage. A tensor fascia lata (TFL) flap was the first choice for trochanteric sores, used in 22 of 25 cases. For ischial sores, the most frequent coverage should have been a gluteus maximus flap, but this muscle was actually used in only four of eight cases. Indeed, most of the patients had already undergone a prior surgery, and we had to use unusual flaps, such as a biceps femoris flap in five cases and a vastus lateralis flap in two cases.

Bacteriology. Multiple bone biopsies and deep tissue samples were obtained during surgery. The bacteriological analysis remained negative in four cases of SH. For the other bone samples, the distribution of the bacteriological species is shown in Figure 4. The most frequent microbe was Staphylococcus aureus, but the proportion of sepsis due to Gram-negative species was also substantial. Antibiotic treatment was started immediately after surgery and was secondarily adapted to the

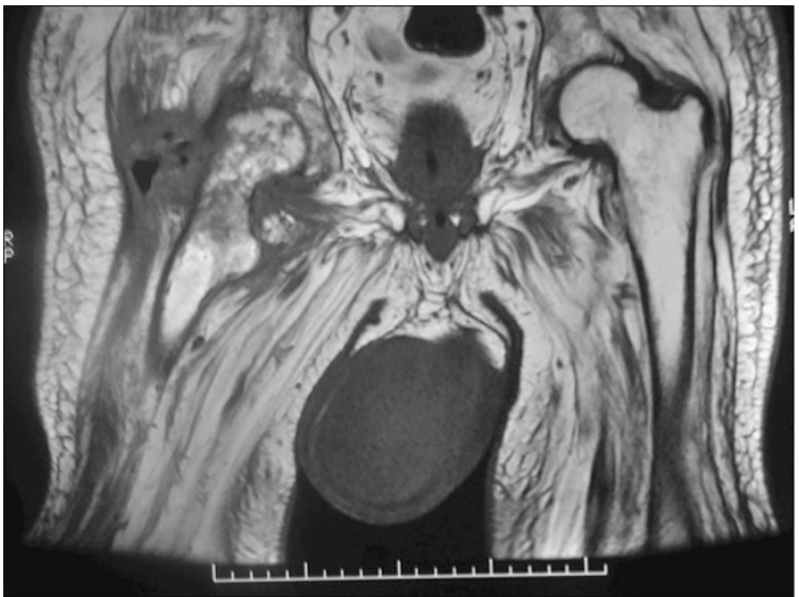

Figure 2 Change of the MRI signal due to a sepsis of the right hip.

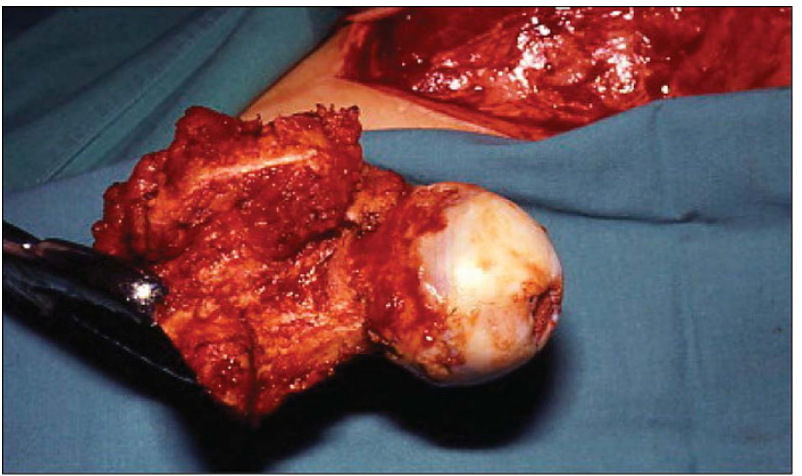

Figure 3 Proximal femoral resection. 


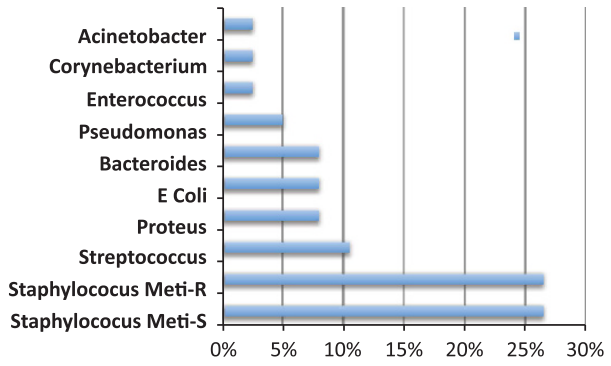

Figure 4 Distribution of the peroperative bacteriological results.

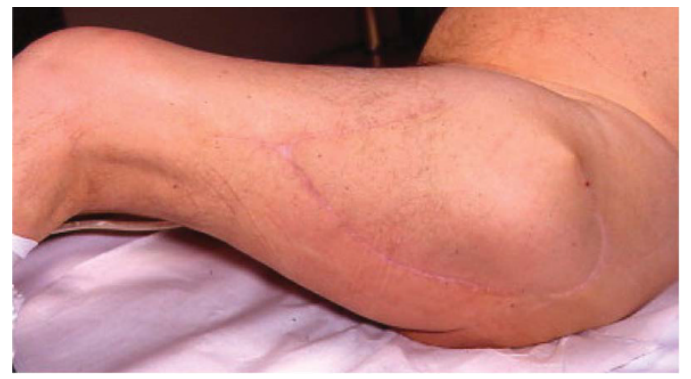

Figure 5 Healing after the postoperative period of 6 weeks.

bacteriological results. A combination of two targeted antibiotics was usually prescribed and was continued for 3 months.

\section{Postoperative complications and their management}

In 19 of the $33 \mathrm{SH}$ cases, we reported no complication during this healing period. In six cases, minor complications occurred, such as small dehiscence of the suture line and/or hematoma. In five patients, a severe early septic complication led to an immediate new cleaning surgical procedure, and in three patients, remaining chronic sepsis within the first 6 postoperative months required a second delayed surgery. At discharge from the PRM department, the wound was always completely healed (Figure 5) and all the patients were able to sit in their wheelchairs with dangling hips. The quality of the patient's position on his cushion was drastically evaluated and corrected. The mean length of stay in the hospital was 9.05 months (1.5-33; s.d. =2.8). The longest stays were due to the most severe complications and/or multiple surgical procedures, but even after exclusion of the 12 stays longer than 9 months, the mean length of hospitalization remained 6.1 months.

\section{DISCUSSION}

The incidence of SH due to pelvic pressure sores is not clearly known, but it is likely high. A previous study by our French SCI referral center found an incidence of approximately $17.5 \%$ in 118 flaps for pelvic pressure sores, ${ }^{3}$ and Michaelis described 9 cases in 3000 patients treated in Stoke-Mandeville. ${ }^{3}$ A complete thoracic level was the preeminent clinical presentation in the current study, which was already noted by Klein ${ }^{4}$ and Byrne. ${ }^{1}$ When patients are functionally very dependent, the risk of pressure sores is most likely linked to the educational level of both the patient and the caregivers rather than to the neurological level itself. Psychiatric disorders ${ }^{5}$ are also known to be major risks factors, and they can contribute to a lack of follow-up that could also be, in our opinion, a primary determinant of severity. The combination of risk factors and management of the various complications resulted in long hospital stays. A typical case is described in Box 1. Inadequate follow-up predisposed to an increased number of

\section{Box 1 A typical case-report}

Mr PA was 23 years old in August 2003 when he was injured in a moped accident resulting in AIS A T9 flaccid paraplegia. Initial rehabilitation lasted 4 months and included therapeutic education concerning prevention of skin breakdown. In March 2005, the patient, who had never attended the planned systematic follow-up visits, presented with a left ischial pressure sore that had to be treated surgically with an uneventful postoperative course. In December 2005, Mr PA consulted for two ischial pressure sores that were also treated surgically. Preventive management of skin breakdown was again reviewed. Immediately after discharge, Mr PA was lost to medical and social (notably employment) follow-up. In January 2007, the patient contacted us to report a state of generalized weakness and an ambulance was sent to his home. On arrival, he presented four pelvic pressure sores (two trochanteric, one right ischial and one sacral) associated with severe malnutrition. Pressure sores were initially treated medically, but in March 2007 Mr PA discharged himself from the PRM department before complete healing. On 2 July 2007, Mr PA was readmitted to our department via the emergency room with 11 pelvic and lower limb pressure sores, he presented no significant peripheral artery disease. The left trochanteric pressure sore, which had not healed at the time of the previous discharge from hospital, had a suspicious appearance and MRI of the pelvis confirmed the presence of $\mathrm{SH}$, which was treated according to our usual practice. The subsequent course was favorable. The right trochanteric pressure sore was treated surgically in June 2008. In August 2008, the patient developed extensive deep vein thrombosis in one leg. During this hospitalization, Mr PA also experienced hypomanic decompensation of a psychiatric illness, leading to introduction of treatment with a mood regulator and organization of psychiatric follow-up. The complete preventive assessment of paraplegia was also performed during this admission, as it had not been performed on an outpatient basis since the initial period and while observing healing times. More appropriate housing was obtained and the patient resumed contact with our employment unit. Stricter follow-up was then organized, but a call from his home nurse led to readmission between March and November 2009 for surgical management of a left ischial pressure sore. Mr PA was found dead at home on 7 May 2010, with no cause of death brought to our knowledge.

concomitant pressure sores and thus of associated treatments, as well as delayed diagnosis of $\mathrm{SH}$ with even bilateral SH. A complete re-assessment of paraplegia was performed and other organ complications had to be treated and high-risk situations, especially neurourological, had to be controlled. Elements of prevention had to be re-explained to the patient, together with more intensive social support. Evans ${ }^{2}$ noted that the diagnosis of $\mathrm{SH}$ was often missed; before proximal femoral resection, $67 \%$ of 15 patients in his study underwent attempted flap closure for pressure sores, which always failed. Peters ${ }^{6}$ also reported a total of 25 procedures for pressure ulceration in 9 patients before the femurectomy. These reports should constitute a definitive argument in favor of highly specialized management and follow-up in SCI referral centers. Any trochanteric or ischial pressure sore with persistent drainage must be considered SH until proven otherwise, ${ }^{2}$ but our actual experience shows that this conclusion is insufficiently supported. Furthermore, we do not clearly know the mortality of patients with SCI due to these undiagnosed complications. Indeed, severe clinical alterations in patients linked to this septic context have also been described in the literature, and they could lead to hospitalization in an intensive care unit. $^{7}$

Authors ${ }^{2,7,8,9}$ who have discussed this surgical treatment have mostly agreed with the necessity of large excision of the necrotic tissues and of an upper-quarter femurectomy based on a modified Girdlestone's procedure ${ }^{10}$ with extended resection to the level of healthy and bleeding bone. ${ }^{6,11}$ This proximal resection is associated 
with closure with a musculocutaneous flap. This procedure is a much less traumatic therapy than disarticulation of a lower limb, ${ }^{12}$ which should only be indicated in patients with severe and recurrent sores with no alternative treatments remaining. ${ }^{13-15}$ There might nevertheless be discrepancies in the literature regarding the therapeutic management.

First, there have been discussions whether the surgical procedure should be performed in one or two stages. Klein ${ }^{4}$ recommended a procedure in two stages, with an interval of between 4 and 11 weeks and the closure time thus being delayed until healthy granulation tissue is obtained. By contrast, our belief, also defended by Evans, ${ }^{2}$ is that treatment in a one-stage procedure does not increase the rate of postoperative complications. Large, opened cavities can be subsequently more difficult to sterilize, and Benito-Ruiz ${ }^{13}$ also commented that the muscle provides vascularization, hastens healing and allows antibiotics to reach the scarred tissue. In Berlemont's study, ${ }^{8} 103$ of 170 operations were performed in one stage, and severe acute situations seemed to constitute the indications for two-stage procedures.

Second, the modality of the flap should be guided by the localization of the causal pressure sore, ${ }^{16-18}$ and we mainly preferred the gluteus maximus muscle for ischial pressure sores and the TFL for trochanteric pressure sores. Our preference contrasted with that of other authors, ${ }^{16}$ who used Minami's procedure, ${ }^{19}$ which employs a vastus lateralis flap. The main drawback of the latter choice for firstline surgery is the sacrifice of the TFL, which thus cannot be reused in the case of later recurrence. ${ }^{9}$ We used a vastus lateralis flap only after a medicosurgical multidisciplinary discussion in cases of the previous surgical treatment of pressure sores, the recurrence of which led us to choose unusual flaps.

Third, some teams ${ }^{4,16}$ have also advocated filling of the resection space with the vastus lateralis muscle to avoid a chronic collection that can become secondarily infected. However, Lortat-Jacob ${ }^{7}$ also described muscle transposition into the empty space that was secondarily complicated by an abscess. In our procedure, this risk was lessened by closed suction with multiple drains.

Finally, another issue involved the postoperative immobilization. Bonded skeletal traction and motion control of the lower limbs, eventually associated with antispasmodic medications, are, in our opinion, sufficient to prevent the femoral shaft pistoning that incurs the risks of large amounts of exudation or bleeding, subsequent flap suffering and secondary heterotopic ossifications of the resection cavity. ${ }^{11}$ We also believe that this traction prevented complications due to pelvic-femoral external fixators on frequently osteopenic bone. ${ }^{4,7,9}$ When a patient had to be moved during the prone period, the recently operated hip had to be maintained in extension. This extension required the synchronized intervention of at least two welltrained caregivers, who also had to verify the adequate position of the patient on his bed several times per day. After healing, phased flexion postures of the hip progressively stretched the skin of the operated zone and helped prevent functional consequences.

The bacteriological diagnosis of $\mathrm{SH}$ was based on perioperative biopsies (femur, acetabulum, joint capsule and soft tissues). Even if $S$. aureus dominated our results, Gram-negative species were often involved. This finding was explained by the colonization of the pelvic area with bacteria from the urogenital and digestive tracts. Similar observations have also been noted by other authors, ${ }^{4,16}$ who have reported the constant growth of more than one microorganism. Multiple deep surgical samples must be acquired to target the choice of antibiotics in a convenient manner. The first postoperative antibiotic treatment was a blind treatment, based on bacteriological probability, and secondarily adjusted to the bacterial spectrum. Four cultures remained negative, perhaps because of prior frequent antibiotic treatments, and the probabilistic choice was maintained during the same period for at least three of the patients. No uninformed empirical antibiotic therapy should be established before surgery. This prohibition leads to an important but unclear choice during the preoperative period between a treatment based on prior superficial samples $^{7}$ and a 2 -week period with no antibiotic medication, which is our common practice. An argument appearing in Heym's study ${ }^{20}$ concerning pressure sore surgery showed that only $36 \%$ of presurgical samples were in good agreement with the surgical samples in 77 patients.

\section{CONCLUSION}

The gold standard treatment for SH after SCI is, in our opinion, a one-stage procedure, including a Girdlestone's procedure and a musculocutaneous flap, with suction drainage but no external fixator, associated with close motion control of the lower limbs. The antibiotic treatment must be adapted to deep samples obtained during surgery. Personal risk factors must be controlled as much as possible, and we are also persuaded that the organizational environment for the primary management and, subsequently, the systematic follow-up of these patients in referral centers can have a major impact on prevention. And preventive measures should definitely be considered as keystones of the policy toward these complications, from medical, social and economic points of view.

\section{DATA ARCHIVING}

There were no data to deposit.

\section{CONFLICT OF INTEREST}

The authors declare no conflict of interest.

1 Byrne DW, Salzberg CA. Major risk factor for pressure ulcers in the spinal cord disabled literature review. Spinal Cord 1996; 34: 255-263.

2 Evans GRD, Lewis VL, Manson PN, Loomis M, Vander Kolk CA. Hip joint communication with pressure sore: the refractory wound and the role of Girdlestone arthroplasty. Plast Reconstr Surg 1993; 91: 288-294.

3 Perrouin-Verbe B, Poirier P, Rome J, Touchais S, Le Fourn B, Lejeune F et al. Hip arthritis in spinal cord injuries patients. Colin D, Barrois B, Pélissiér J. L'escarre. Masson: Paris, 1998, pp. 176-183.

4 Klein N, Moore Th, Capen D, Green S. Sepsis of the hip in paraplegic patients. J Bone Joint Surg 1988; 70-A: 839-843.

5 Heilporn A. Psychological factors in the causation of pressure sores: case reports. Paraplegia 1991; 29: 137-139.

6 Peters JW, Johnson GE. Proximal femurectomy for decubitus ulceration in the spinal cord injury patient. Paraplegia 1990; 28: 55-61.

7 Lortat-Jacob A, Lortat-Jacob S, Jouanin Th, Beaufils Ph, Coignard S, Held JP et al. Septic arthritis of the hip in paraplegics. Rev Chir Orthop (Paris) 1984; 70: 383-388.

8 Berlemont M. The treatment of suppurative arthritis of the hip in paraplegia by extensive hip resection. Rev Chir Orthop (Paris) 1985; 71: 377-387.

9 Klein N, Luster S, Green S, Moore Th, Capen D. Closure of defects from pressure sores requiring proximal femoral resection. Ann Plast Surg 1988; 21: 246-250.

10 Girdlestone GR. Acute pyogenic arthritis of the hip: an operation giving free access and effective drainage. 1943. Clin Orthop Relat Res 2008; 466: 258-263.

11 Rubayi S, Pompan D, Garland D. Proximal femoral resection and myocutaneous flap for treatment of pressure ulcers in spinal cord injury patients. Ann Plast Surg 1991; 27: $132-138$.

12 Lawton RL, De Pinto V. Bilateral hip disarticulation in paraplegics with decubitus ulcers. Arch Surg 1987; 122: 1040-1043.

13 Benito-Ruiz J, Baena-Montilla P, Mena-Yago A, Miguel I, Montanana-Vizcaino J. A complicated trochanteric pressure sore: what is the best surgical management? Case report. Paraplegia 1993; 31: 119-124.

14 Vancabeke M, Harper L, Penders W, Putz P. Anterior flap for coverage following hip disarticulation for osteomyelitis. Acta Orthop Belg1999; 65: 223-225.

15 Correa GI, Calderon WO, Roa RG, Guzman LM, Burnier LA, Danilla SE. Proximal amputation of inferior extremity secondary to recurrent pressure ulcers in patients with spinal cord injuries. Spinal Cord 2008; 46: 135-139. 
16 Acartürk TO. Treatment of large ischial ulcers communicating with the hip joint with proximal femoral resection and reconstruction with a combined vastus lateralis, vastus intermedius and rectus femoris musculocutaneous flap. J Plast Reconstr Aesthet Surg 2009; 62: 1497-1502.

17 Sagi A, Meller Y, Kon M, Rosenberg L, Ben-Yakar Y. Bilateral hip resection for closure of trochanteric pressure sores: case report. Paraplegia 1987; 25: 39-43.
18 Tryggestad KE, Youm T, Koval KJ. Orthopedic management of decubitus ulcers around the proximal femur. Am J Orthop (Belle Mead NJ) 2006; 35: 316-321.

19 Minami RT, Hentz VR, Vistnes LM. Use of the vastus lateralis muscle flap for repair of trochanteric pressure sores. Plast Reconstr Surg 1977; 60: 364-368.

20 Heym B, Rimareix F, Lortat-Jacob A, Nicolas-Chanoine MH. Bacteriological investigation of infected pressure ulcers in spinal cord-injured patients and impact on antibiotic therapy. Spinal Cord 2004; 42: 230-234. 